\title{
Research Article \\ Shock in the Yarn during Unwinding from Packages
}

\author{
Stanislav Praček, Klemen Možina, and Franci Sluga \\ Department of Textiles, NTF, University of Ljubljana, Snežniška 5, 1000 Ljubljana, Slovenia \\ Correspondence should be addressed to Stanislav Praček; stane.pracek@ntf.uni-lj.si
}

Received 14 December 2012; Revised 18 January 2013; Accepted 4 February 2013

Academic Editor: Lan Xu

Copyright (C) 2013 Stanislav Praček et al. This is an open access article distributed under the Creative Commons Attribution License, which permits unrestricted use, distribution, and reproduction in any medium, provided the original work is properly cited.

\begin{abstract}
Tension in the yarn and its oscillations during the over-end unwinding of the yarn from stationary packages depend on the unwinding speed, the shape and the winding type of the package, the air drag coefficient, and also the coefficient of friction between the yarn and the package. The yarn does not leave the surface package immediately at the unwinding point. Instead, it first slides on the surface and then lifts off to form the balloon. The problem of simulating the unwinding process can be split into two smaller subproblems: the first task is to describe the motion of the yarn in the balloon; the second one is to solve the sliding motion. In spite of the seemingly complex form of the equations, they can be partially analytically solved as we show in the paper.
\end{abstract}

\section{Introduction}

During the yarn unwinding from a stationary package, the yarn slides on the surface of the package before it lifts off to form a balloon. The point where the yarn begins to slide is known as the unwinding point, while the point where the yarn lifts off from the surface is known as the lift-off point. On this section of the yarn, that is, between the unwinding point and the lift-off point, the tension in the yarn drops from its value in the balloon (at the lift-off point) to its residual value, defined as the tension of the yarn inside the package. The equations of motion which govern the motion of the yarn are known: we have established them in Section 2 of this paper. They can be partially analytically solved, as we show in the following. The theory of yarn unwinding off a package and the balloon theory had a quick development in the fifties because of Padfield's work [1,2]. She fixed Mack equations for the balloon [3] so that they take into account the Coriolis system force. She found the results for a single balloon as it unwinds from a cylindrical package. The same theory was later used to calculate the parameters for multiple consecutive balloons with a nonzero unwinding angle and a cylindrical, conical, or empty package [1]. Kothari and Leaf derived motion equations that include the effect of the gravity force and air resistance force tangential component $[4,5]$. Using extensive numerical methods for cylindrical and conical packages they showed that these effects can be ignored. Recently Fraser used the motion theory to show that the time dependence can be excluded from motion equations in a mathematical correct way $[6,7]$. He derived movable boundary conditions for packages with small winding angle. Fraser also determined that the tension inside and the radius of a balloon are smaller for an elastic yarn. Using simple physics He recently introduced different nanophenomena in nanotextile that are the newest additions to the theory of electrospinning $[8,9]$.

\section{The Equation of Motion for Yarn}

The problem of yarn motion on the package surface during the unwinding can be treated in analogy with the motion of the yarn forming the balloon between the lift-off point and the eyelet, through which the yarn is being pulled.

The yarn is being withdrawn with velocity $V$ through an eyelet, where we also fix the origin $O$ of our coordinate system (Figure 1). The yarn is rotating aroun the $z$-axis with an angular velocity $\omega$. At the lift-off point Lp, the yarn lifts from the package and forms a balloon. At the unwinding point $U p$, the yarn starts to slide on the surface of the package. Angle $\phi$ is the winding angle of the yarn on the package. 


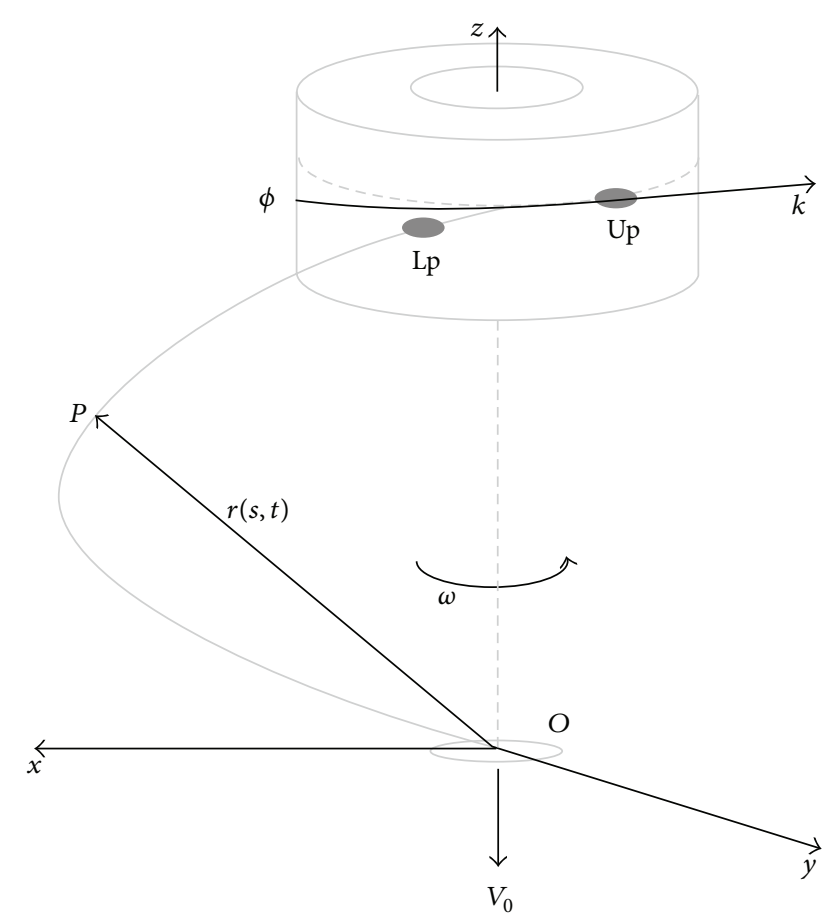

Figure 1: Mechanical setup in over-end yarn unwinding from cylindrical package.

The general equation of motion for the yarn was derived and justified in one of the previous works [10]:

$$
\rho\left(D^{2} r+2 \omega \times D r+\omega \times(\omega \times r)+\dot{\omega} \times r\right)=\frac{\partial}{\partial s}\left(T \frac{\partial r}{\partial s}\right)+f .
$$

The position vector $r$ points from the origin of the coordinate system to a chosen point along the yarn, $\rho$ is the linear density of the yarn mass, $\omega$ is the angular velocity vector of the spinning coordinate system in which the yarn is being described and which points along the $z$-axis, $D$ is the operator of the total time derivative which follows the motion of the point inside the spinning coordinate system, $D=$ $\partial /\left.\partial t\right|_{r, \theta, z}-V \partial / \partial s, T$ is the mechanical tension, and $f$ is the linear density of external forces.

\section{Friction between the Yarn and the Package Surface}

There is a friction between the package and the yarn which is sliding on its surface before it lifts off to form the balloon. The yarn is exerting a normal force on the package (i.e., a force perpendicular to the package surface, thus in radial direction). This force is not known a priori, but must be determined as part of the solution to the full problem. The simplest expression of the friction law states that the friction force is proportional to the normal component of the force. The coefficient of proportionality is known as the coefficient

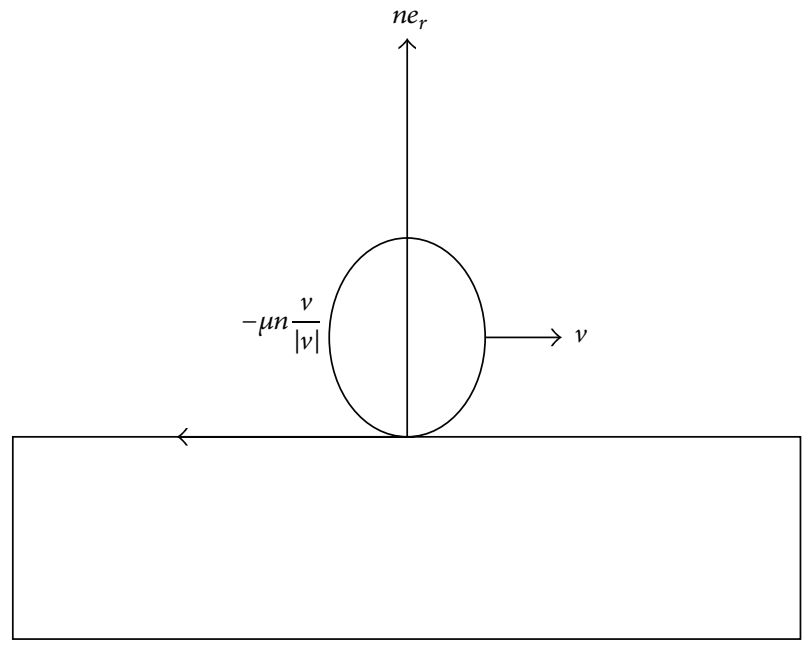

FIGURE 2: The force of friction between the package surface and the yarn.

of friction $\mu$. The friction force points in the direction opposite to the yarn motion.

The quantity $f$ in (1) therefore has two components: the radial force of the package on the yarn (which is equal in magnitude to the force of the yarn on the package, in accordance with Newton's law of reciprocal action) and the friction force proper (Figure 2):

$$
f=n e_{r}-\mu n \frac{v}{|v|} .
$$

Here $n$ is the linear density of the normal component of the force between the yarn and the surface, $e_{r}$ is the unit vector in the radial direction, and $v /|v|$ is the unit vector in the direction of the yarn.

When the yarn slides on the surface, it thus experiences the normal force $n e_{r}$ and the friction force $-\mu n v /|v|$.

The friction law is at best a rough approximation to a more complex real behavior. In reality, the coefficient of friction depends in a complicated way on the sliding velocity [11-16], and it is different at various points of the package surface since the package is seldom fully homogenous. We thus take $\mu$ to be some average coefficient of friction which one can determine empirically [17].

\section{Quasi-Stationary Approximation}

Equation (1) is generally valid and describes an arbitrary motion of the yarn, even in cases when the conditions are rapidly changing, for example, near the package edges. Near the package edge the winding angle suddenly changes, therefore the motion of the yarn on the package surface and in the part of the balloon near the lift-off point becomes very complex. Near the edges, undesired events can occur: the yarn can fall off the package or a layer of the yarn collapses. The description of such transient effects is beyond the validity of our simplified model, since one should accurately model the behavior of the yarn also in the layers forming the package 
bulk. For example, the residual forces of the yarn in the package would also play a role [18].

Strictly speaking, the yarn undergoes sliding motion on the package surface only when the unwinding point is at a certain distance away from the package edges. In such circumstances, the conditions are quasi-stationary: in the rotating coordinate frame the yarn only slowly changes its form. For this reason, in the first approximation the time dependence can be fully described by time-variable boundary conditions, while the time-derivative terms in the equation of motion can be neglected:

$$
\rho\left(V^{2} \frac{\partial^{2} r}{\partial s^{2}}-2 V \omega \times \frac{\partial r}{\partial s}+\omega \times(\omega \times r)\right)=\frac{\partial}{\partial s}\left(T \frac{\partial r}{\partial s}\right)+f
$$

\section{The Equation of Motion for the Yarn on the Package: Simplification to a Two-Dimensional Problem}

When the yarn slides on the package surface, its motion effectively occurs within a two-dimensional subspace. This fact can be taken into account in (3) in order to simplify the problem to a two-dimensional problem which can be handled more easily. It turns out that in the case of sliding motion on the cylindrical package, the problem can be solved to a large extent using analytical techniques. Analytical solutions allow for a more direct understanding of the relation between the different quantities. For this reason, we will henceforth assume that the package is cylindrical, and we will determine the analytical solution.

The radius vector to a point on the surface of a cylinder can be expressed as (compare with equation (17) in [10])

$$
r(s)=c e_{r}(\theta(s))+z(s) e_{z}
$$

The quantity $c$ is the constant distance of the point $r$ from the package axis. It is equal to the radius of the layer which is being unwound. The unit vector $e_{z}$ points along the direction of the package axis, and the unit vector $e_{r}$ points in the radial direction with the polar angle $\theta(s)$ (see Figure 3 ). There are two unknowns in this expression, $\theta(s)$ and $z(s)$, while the third $[r(s)]$ drops out since it is constant on the surface. The motion of the yarn has thus been translated to a twodimensional problem. This ansatz will be used in (4) to find a simplified equation of motion.

The arc-length derivatives of the radius vector are computed using the relations (18) from [10] to obtain

$$
\begin{gathered}
r^{\prime}(s)=c \theta^{\prime}(s) e_{\theta}+z^{\prime}(s) e_{z}, \\
r^{\prime \prime}(s)=c \theta^{\prime \prime}(s)-c\left[\theta^{\prime}(s)\right]^{2} e_{r}+z^{\prime \prime}(s) e_{z},
\end{gathered}
$$

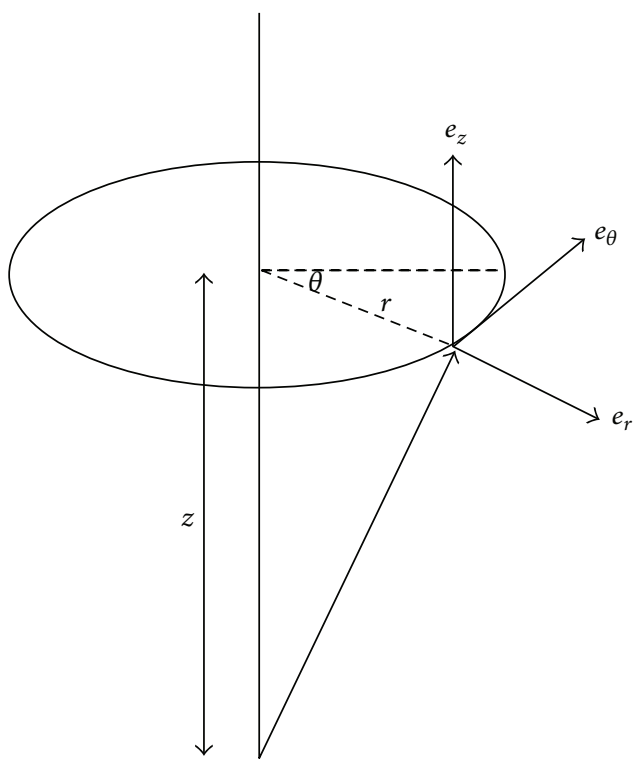

FIGURE 3: The cylindrical coordinate system.

where the dashes indicate the arc-length derivative. We then obtain

$$
\begin{aligned}
& \frac{\partial}{\partial s}\left(T \frac{\partial r}{\partial s}\right) \\
& \quad=\frac{\partial T}{\partial s} \frac{\partial r}{\partial s}+T \frac{\partial^{2} r}{\partial s^{2}} \\
& \quad=T^{\prime}\left(c \theta^{\prime} e_{\theta}+z^{\prime} e_{z}\right)+T\left(c \theta^{\prime \prime} e_{\theta}-c\left(\theta^{\prime}\right)^{2} e_{r}+z^{\prime \prime} e_{z}\right) \\
& \quad=-c T\left(\theta^{\prime}\right)^{2} e_{r}+c\left(T^{\prime} \theta^{\prime}+T \theta^{\prime \prime}\right) e_{\theta}+\left(T^{\prime} z^{\prime}+T z^{\prime \prime}\right) e_{z}
\end{aligned}
$$

We also need the relations

$$
\begin{aligned}
& \omega \times r^{\prime}=-c \omega \theta^{\prime}(s) e_{r}, \\
& \omega \times(\omega \times r)=-\omega^{2} c e_{r}
\end{aligned}
$$

which can be derived using a simple calculation of the vector products.

Equation (3) may then be decomposed along its different components:

$$
\begin{gathered}
(r) \rho\left(-c V^{2}\left(\theta^{\prime}\right)^{2}+2 V c \omega \theta^{\prime}-\omega^{2} c\right)=-c T\left(\theta^{\prime}\right)^{2}+f_{r}, \\
(\theta) \rho\left(c V^{2} \theta^{\prime \prime}\right)=c T \theta^{\prime \prime}+c T \theta^{\prime}+f_{\theta} \\
(z) \rho\left(V^{2} z^{\prime \prime}\right)=T z^{\prime \prime}+T z^{\prime}+f_{z} .
\end{gathered}
$$

The quantities $f_{r}, f_{\theta}$, and $f_{z}$ are the components of the linear density of the external force (2). The first one is simply $f_{r}=n$, while the other two still need to be determined. The velocity of the yarn in the quasi-stationary approximation is (see equation (23) in [10], where we substitute $v_{\text {rel }}=0$ )

$$
v=-V t+\omega \times r=c\left(\omega-V \theta^{\prime}\right) e_{\theta}-z^{\prime} V e_{z} .
$$


This expression can then be used to derive the unit vector in the direction of the yarn velocity:

$$
\frac{v}{|v|}=\frac{1}{\sqrt{c^{2}\left(\omega-V \theta^{\prime}\right)^{2}+z^{\prime 2} V^{2}}}\left[c\left(\omega-V \theta^{\prime}\right) e_{\theta}-z^{\prime} V e_{z}\right],
$$

from which then finally follow the two components of the linear density of the force:

$$
\begin{aligned}
& f_{\theta}=\frac{-\mu n c\left(\omega-V \theta^{\prime}\right)}{\sqrt{c^{2}\left(\omega-V \theta^{\prime}\right)^{2}+z^{\prime 2} V^{2}}}, \\
& f_{z}=\frac{\mu n z^{\prime} V}{\sqrt{c^{2}\left(\omega-V \theta^{\prime}\right)^{2}+z^{\prime 2} V^{2}}} .
\end{aligned}
$$

Equations (8)-(10) and (13) are the simplified equations of motions that we required. At first they appear more complex than the vector expressions (2) and (3), since they are expressed component by component. Nevertheless, they are indeed simpler: the unknown functions are $\theta, z, n_{\theta}, n_{z}$, and $T$, but we have managed to eliminate $r$ and $n_{r}$. In this part of the paper we will show that the function $T$ can equally be eliminated.

\section{Partial Analytical Solution}

Equation (9) from the previous section is multiplied by $c \theta^{\prime}$, (10) by $z^{\prime}$; they are then added together and reorganized to read

$$
\begin{aligned}
\rho V^{2}\left(c^{2} \theta^{\prime} \theta^{\prime \prime}+z^{\prime} z^{\prime \prime}\right)= & T\left(c^{2} \theta^{\prime} \theta^{\prime \prime}+z^{\prime} z^{\prime \prime}\right) \\
& +T^{\prime}\left(c^{2} \theta^{\prime 2}+z^{\prime 2}\right)+c \theta^{\prime} f_{\theta}+z^{\prime} f_{z} .
\end{aligned}
$$

In this equation, $\rho$ is the linear density of the yarn, $V$ the unwinding velocity, $c$ the package radius, $T$ the tension in the yarn, $f$ the linear density of the force of friction, and the position of the point is given in the cylindrical coordinate system $(r \theta z)$. The dash after a symbol denotes the operation of taking the derivative with respect to the arc length $s$. Now we take into account the condition of nonextensibility, which states that the extension of yarn may be neglected. For motion on the package surface, this condition (equation (34) in [10]) can be expressed as

$$
c^{2} \theta^{\prime 2}+z^{\prime 2}=1
$$

Taking a derivative of this equation, we obtain

$$
c^{2} \theta^{\prime} \theta^{\prime \prime}+z^{\prime} z^{\prime \prime}=0
$$

Inserting (15) and (16) into (14), we end up with

$$
T^{\prime}=-c \theta^{\prime} f_{\theta}-z^{\prime} f_{z}
$$

In this equation we insert the expressions for the components of the linear density of the force

$$
\begin{aligned}
& f_{\theta}=\frac{-\mu n c\left(\omega-V \theta^{\prime}\right)}{\sqrt{c^{2}\left(\omega-V \theta^{\prime}\right)^{2}+z^{\prime 2} V^{2}}}, \\
& f_{z}=\frac{\mu n z^{\prime} V}{\sqrt{c^{2}\left(\omega-V \theta^{\prime}\right)^{2}+z^{\prime 2} V^{2}}}
\end{aligned}
$$

and we obtain

$$
\begin{aligned}
T^{\prime} & =\frac{\mu n\left(c^{2} \theta^{\prime}\left(\omega-V \theta^{\prime}\right)-z^{\prime 2} V\right)}{\sqrt{c^{2}\left(\omega-V \theta^{\prime}\right)^{2}+z^{\prime 2} V^{2}}} \\
& =\frac{\mu n\left(c^{2} \omega \theta^{\prime}-V\right)}{\sqrt{c^{2}\left(\omega-V \theta^{\prime}\right)^{2}+z^{\prime 2} V^{2}}} .
\end{aligned}
$$

To obtain the last expression we have used (15). The $n$ from this equation is evaluated and inserted in the expression for $f_{\theta}$ in (18):

$$
f_{\theta}=c T^{\prime} \frac{V \theta^{\prime}-\omega}{c^{2} \omega \theta^{\prime}-V} .
$$

This is then used in

$$
\rho c V^{2} \theta^{\prime \prime}=c T \theta^{\prime \prime}+c T^{\prime} \theta^{\prime}+f_{\theta}
$$

to obtain

$$
\left(\rho V^{2}-T\right) \theta^{\prime \prime}=T^{\prime} \theta^{\prime}+\frac{V \theta^{\prime}-\omega}{c^{2} \omega \theta^{\prime}-V} T^{\prime}=\omega T^{\prime} \frac{c^{2} \theta^{\prime 2}-1}{c^{2} \omega \theta^{\prime}-V}
$$

We rewrite this equation as

$$
\frac{c \omega}{V} \frac{T^{\prime}}{\rho V^{2}-T}=c \theta^{\prime \prime}\left[\frac{\left(c^{2} \omega / V\right) \theta^{\prime}-1}{c^{2} \theta^{\prime 2}-1}\right] .
$$

After introducing the dimensionless angular velocity $\Omega=$ $c \omega / V$ and a new variable $\chi=c \theta$, the equation takes a more clear expression:

$$
\Omega \frac{T^{\prime}}{\rho V^{2}-T}=\chi^{\prime \prime}\left[\frac{1-\Omega \chi^{\prime}}{1-\chi^{\prime 2}}\right] .
$$

The quantity $\chi^{\prime}=c \theta^{\prime}$ is always smaller than 1 when the yarn slides on the package surface, since the length of one loop of yarn on the package is at least $2 \pi c$. A simple consideration (and the help of Figure 4) can convince us that the derivative $\chi^{\prime}$ is related with the tangential direction of the yarn on the package surface. In fact, one has $\chi^{\prime}=\cos \phi$. Using a similar consideration one can also establish that $z^{\prime}=\tan \phi$.

Equation (24) can be integrated analytically. The left hand side is the derivative of the function $-\Omega \ln \left|T-\rho V^{2}\right|$, while the right hand side is the derivate of the function $((\Omega-1) / 2) \ln \mid 1-$ $\chi^{\prime \prime}|+((\Omega+1) / 2) \ln | 1+\chi^{\prime \prime} \mid$. 


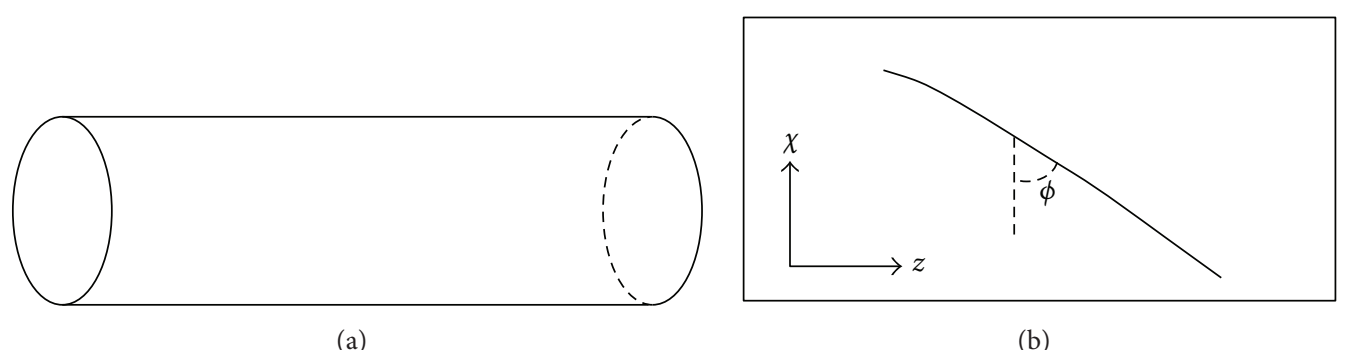

(a)

(b)

Figure 4: (a) The surface of the cylinder is cut along the long edge and the surface is flattened. (b) The flattened surface is a plane with axes $z$ and $\chi$. The angle $\phi$ is the angle of the yarn in the $(z \chi)$ plane.

As can be easily verified, we thus obtain

$$
\begin{array}{r}
-\Omega \ln \left|T-\rho V^{2}\right| \frac{(\Omega-1)}{2} \ln \left|1-\chi^{\prime}\right| \\
+\frac{(\Omega+1)}{2} \ln \left|1+\chi^{\prime}\right|+\text { const. }
\end{array}
$$

The tension $T$ is always larger than the quantity $\rho V^{2}$, which is twice the linear density of the kinetic energy which the yarn has because of it being pulled through the eyelet $[19,20]$. We have also already established that $\chi^{\prime}<1$. For this reason, all quantities between the absolute value brackets are positive, thus the brackets do not need to be written.

Exponentiating the expression we had obtained and rearranging it slightly, we obtain

$$
T-\rho V^{2}=K\left[\left(1-c \theta^{\prime}\right)^{((1-\Omega) / 2 \Omega)}\left(1+c \theta^{\prime}\right)^{-((1+\Omega) / 2 \Omega)}\right],
$$

where $K$ is an integration constant. It can be determined by considering the behavior at the lift-off point. If the winding angle is $\Phi$, then the change of the arc length $s$ by $2 \pi c / \cos \Phi$ (i.e., the length of one loop) corresponds to a change of $\theta$ by $2 \pi$.

Therefore at the lift-off point $\theta^{\prime}$ is equal to $\cos \Phi / c$, and finally $\chi^{\prime}(\mathrm{Od})=\cos \Phi$. (The winding angle $\Phi$ is by definition equal to the angle of the yarn in the $(z \chi)$ plane, therefore this result is in full agreement with the expression $\chi^{\prime}=\cos \phi$ which we had established before.) In this point the tension in the yarn is equal to the residual tension of the yarn inside the package, $T_{\text {res }}$. If both expressions are used in (26), we obtain

$$
T_{\text {res }}-\rho V^{2}=K\left[(1-\cos \Phi)^{((1-\Omega) / 2 \Omega)}(1+\cos \Phi)^{-((1+\Omega) / 2 \Omega)}\right] .
$$

Equation (26) may therefore be written as

$$
\begin{aligned}
& \frac{T-\rho V^{2}}{T_{\text {res }}-\rho V^{2}} \\
& \quad=\left[\left(\frac{1-c \theta^{\prime}}{1-\cos |\Phi|}\right)^{((1-\Omega) / 2 \Omega)}\left(\frac{1+c \theta^{\prime}}{1+\cos |\Phi|}\right)^{-((1+\Omega) / 2 \Omega)}\right] .
\end{aligned}
$$

In parallel cylindrical package with dense parallel winding, the dimensionless angular velocity $\Omega=c \omega / V$ is approximately equal to 1 . Setting $\Omega=1$ in (26) we obtain

$$
T-\rho V^{2}=\frac{K}{1+c \theta^{\prime}} .
$$

This result had already been established by Fraser et al. [6], but our equation (26) holds in general. In cross-wound package one namely has

$$
\Omega=\frac{\cos \Phi}{1-\sin \Phi}
$$

where $\Phi$ is the winding angle at the point where the yarn is currently being unwound. This implies that in cross-wound packages, the dimensionless angular velocity is not equal to one, but it is larger than 1 during the unwinding in the backward direction $(\Phi>1)$ and smaller than 1 during the unwinding in the forward direction $(\Phi<1)$.

In the section of yarn which slides on the surface and experiences friction from the lower layers, the tension decreases from the value at the lift-off point to the residual value. At the same time, the angle $\phi$ increases from its value at the unwinding point to the value of $\Phi$ at the lift-off point. The relation between these two phenomena is given precisely by (28).

Equation (19) can be rewritten as

$$
\begin{aligned}
T^{\prime} & =\frac{\mu n(V \Omega \cos \phi-V)}{\sqrt{V^{2}(\Omega-\cos \phi)^{2}+V^{2} \tan ^{2} \phi}} \\
& =\frac{\mu n(\Omega \cos \phi-1)}{\sqrt{(\Omega-\cos \phi)^{2}+\tan ^{2} \phi}} .
\end{aligned}
$$

Using the approximation of $\Omega=1$ and $\cos \phi \sim 1-\phi^{2} / 2$, $\tan \phi \sim \phi$, we obtain

$$
T^{\prime} \approx \frac{-\mu n \phi}{2} .
$$

The decrease of the tension along the yarn is proportional to the coefficient of friction, as expected. The larger the coefficient of friction is, the shorter is the sliding segment of the yarn. The derivative is also proportional to the angle $\phi$, thus the decrease is larger near the lift-off point where $\phi$ is large, but smaller at the unwinding point where in the case of dense parallel winding the angle $\phi$ is almost equal to zero. 


\section{Conclusion}

We have shown how the equation of motion on the package surface can be obtained from the general equation of yarn motion by considering the force of friction. The external force has two components: the normal force of the package surface and the force of friction. We have described the conditions for the validity of the quasi-stationary approximation which was then used to simplify the equation of motion to a twodimensional problem. We have also shown that the simplification of the equation of motion for the sliding motion of the yarn to a two-dimensional problem makes it possible to establish the main conclusions analytically. We have shown how the section of the yarn which slides on the package surface makes it possible that the tension in the yarn reduces to its residual yarn and how this is related to the form of the sliding yarn. More accurate solutions of the problem can, however, only be obtained using a full numerical solution of the equations using the shooting method $[6,19,20]$. Another very interesting approach for solving the equation of motion for the yarn would be the use of the methods described by JiHuan He. The analytical solution can be obtained using the variational iteration method or the homotopy perturbation method reviewed in $[8,21,22]$.

\section{References}

[1] D. G. Padfield, "The motion and tension of an unwinding thread," Proceedings of the Royal Society A, vol. 245, no. 1242, pp. 382-407, 1958.

[2] D. G. Padfield, "A note on the fluctuations of tension during unwinding," Journal of the Textile Institute Proceedings, vol. 47, no. 5, pp. 301-308, 1956.

[3] A. E. de Barr and H. Catling, Manual of Cotton Spinning, vol. 5, Butterworth, Oxford, UK, 1976.

[4] V. K. Kothari and G. A. V. Leaf, "The unwinding of yarns from packages. Part I: the theory of yarn-unwinding," Journal of the Textile Institute, vol. 70, no. 3, pp. 89-95, 1979.

[5] V. K. Kothari and G. A. V. Leaf, "The unwinding of yarns from packages. Part II: unwinding from cylindrical packages," Journal of the Textile Institute, vol. 70, no. 5, pp. 172-183, 1979.

[6] W. B. Fraser, T. K. Ghosh, and S. K. Batra, "On unwinding yarn from cylindrical package," Proceedings of the Royal Society A, vol. 436, no. 1898, pp. 479-498, 1992.

[7] W. B. Fraser, "Effect of yarn elasticity on an unwinding balloon," Journal of the Textile Institute, vol. 83, no. 4, pp. 603-613, 1992.

[8] J.-H. He, "An elementary introduction to recently developed asymptotic methods and nanomechanics in textile engineering," International Journal of Modern Physics B, vol. 22, no. 21, pp. 3487-3578, 2008.

[9] S. Q. Wang, J.-H. He, and L. Xu, "Non-ionic surfactants for enhancing electrospinability and for the preparation of electrospun nanofibers," Polymer International, vol. 57, no. 9, pp. 10791082, 2008.

[10] S. Praček, "Theory of string motion in the textile process of yarn unwinding," International Journal of Nonlinear Sciences and Numerical Simulation, vol. 8, no. 3, pp. 451-460, 2007.

[11] J. Strnad, Fizika, Prvi Del: Mehanika, Toplota, DMFA, Ljubljana, Slovenia, 1995.
[12] J. A. Roberson and C. T. Crowe, Engineering Fluid Dynamics, Druga Izdaja, Houghton Mifflin Company, Boston, Mass, USA, 1980.

[13] S. Pahor, Uvod v Analiticno Mehaniko, DMFA, Ljubljana, Slovenia, 1995.

[14] J. N. Bronštejn and K. A. Semendjajev, Matematični Priročnik, Tehniška založba Slovenije, Ljubljana, Slovenia, 1994.

[15] T. K. Ghosh, S. K. Batra, and A. S. Murthy, "Dynamic analysis of yarn unwinding from cylindrical packages. Part I: parametric studies of the two-region problem," Textile Research Journal, vol. 71, no. 9, pp. 771-778, 2001.

[16] T. K. Ghosh, S. K. Batra, and X. Murthy, "Dynamic analysis of yarn unwinding from cylindrical packages. Part II: the threeregion analysis," Textile Research Journal, vol. 71, no. 10, pp. 855861, 2001.

[17] R. Wu, J. Yu, C. D. Rahn, and B. C. Goswami, "Measuring yarn/package friction during over-end unwinding," Textile Research Journal, vol. 70, no. 4, pp. 321-327, 2000.

[18] J. D. Clark, W. B. Fraser, and D. M. Stump, "Modelling of tension in yarn package unwinding," Journal of Engineering Mathematics, vol. 40, no. 1, pp. 59-75, 2001.

[19] X. M. Kong, Steady state unwinding of yarn from cylindrical packages: theory and experiment [Ph.D. thesis], Clemson University, Clemson, SC, USA, 1997.

[20] X. M. Kong, C. D. Rahn, and B. C. Goswami, "Steady-state unwinding of yarn from cyclindrical packages," Textile Research Journal, vol. 69, no. 4, pp. 292-306, 1999.

[21] J.-H. He, "Some asymptotic methods for strongly nonlinear equations," International Journal of Modern Physics B, vol. 20, no. 10, pp. 1141-1199, 2006.

[22] J.-H. He, "Asimptotic methods for solitary solutions and compactons," Abstract and Applied Analysis, vol. 2012, Article ID 916793, 130 pages, 2012. 


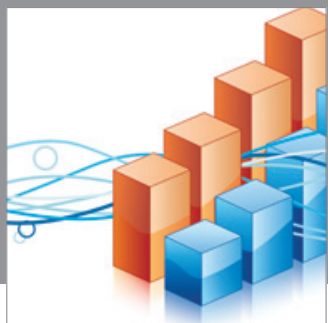

Advances in

Operations Research

mansans

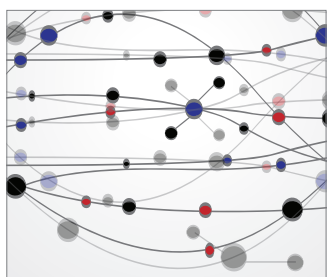

The Scientific World Journal
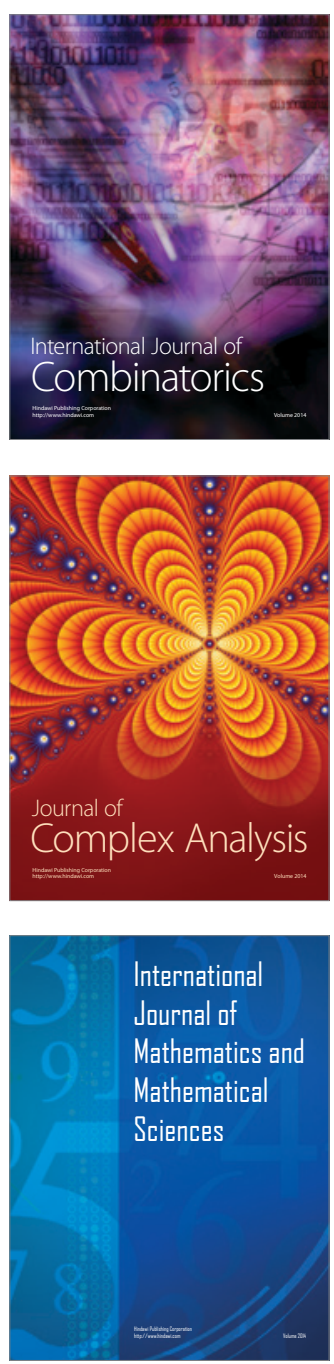
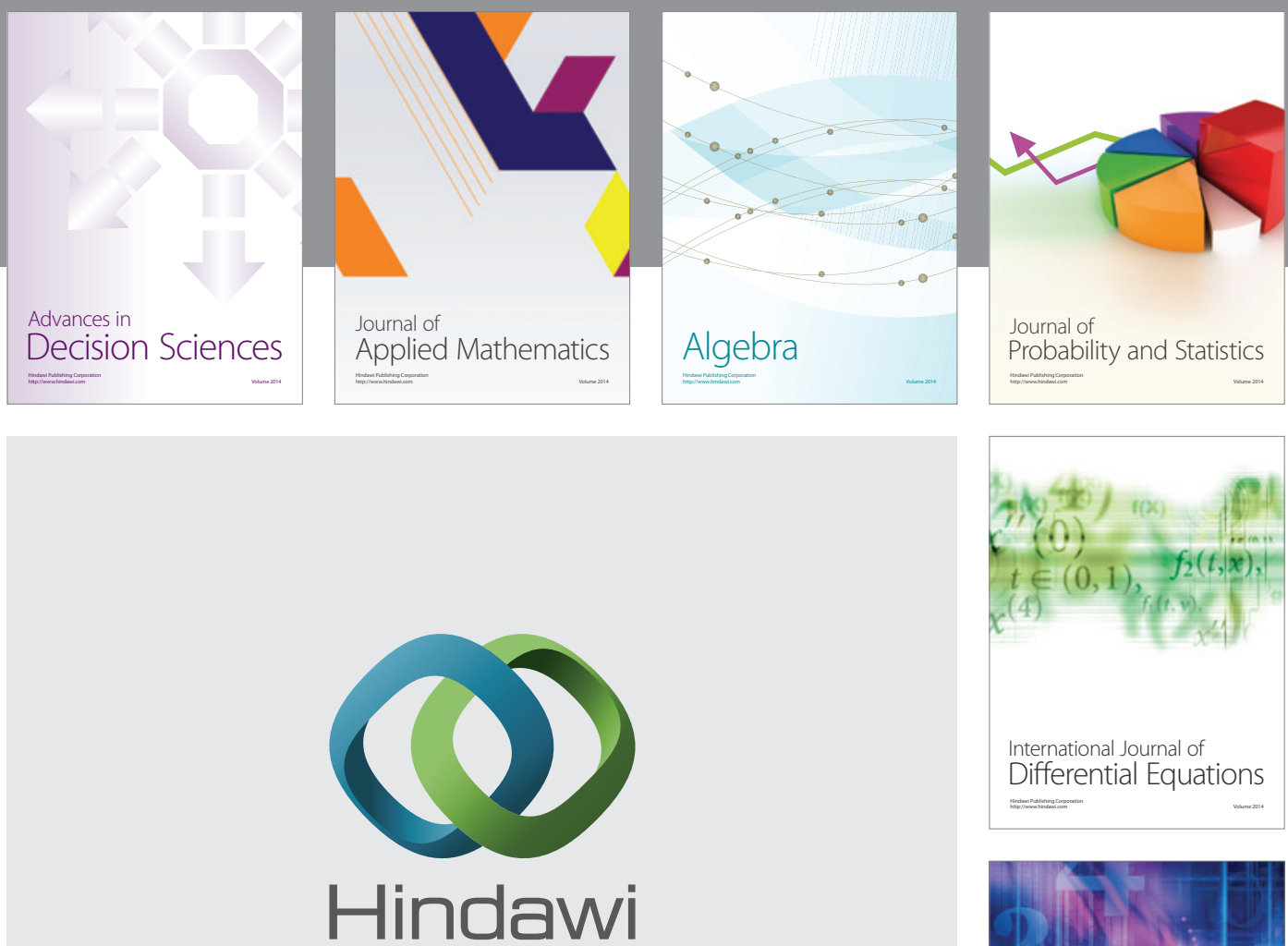

Submit your manuscripts at http://www.hindawi.com
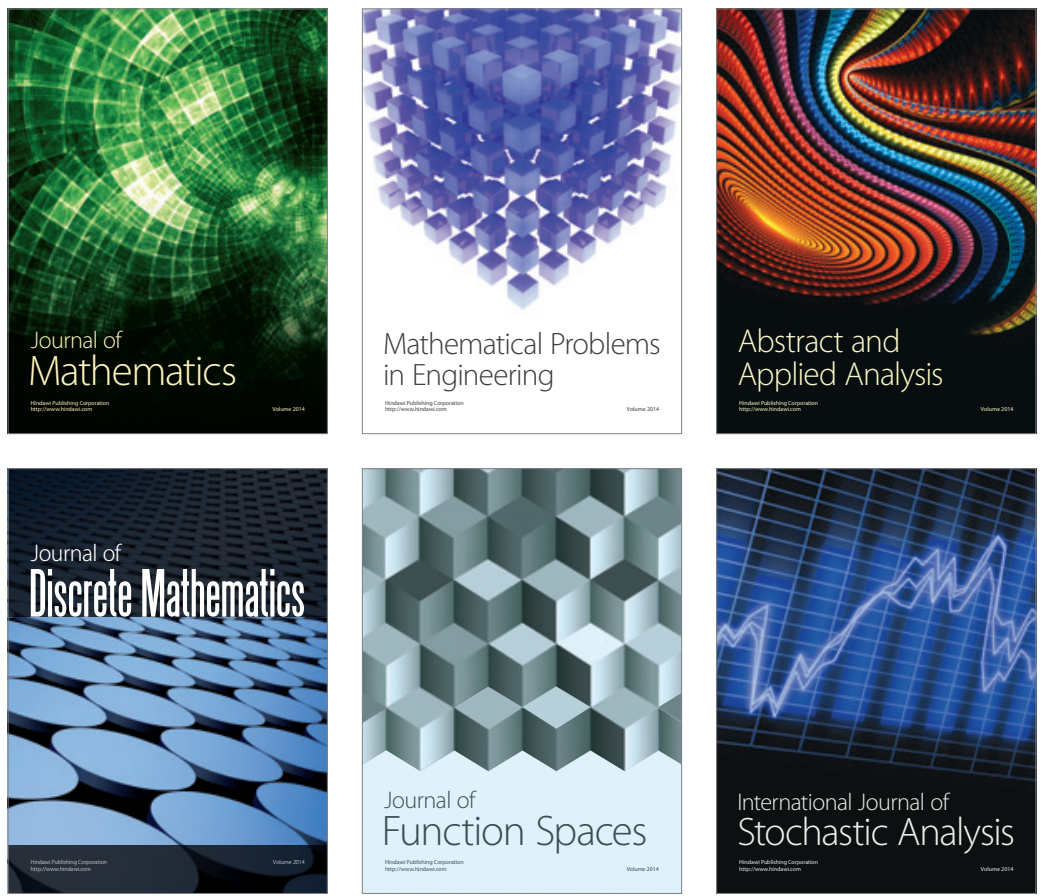

Journal of

Function Spaces

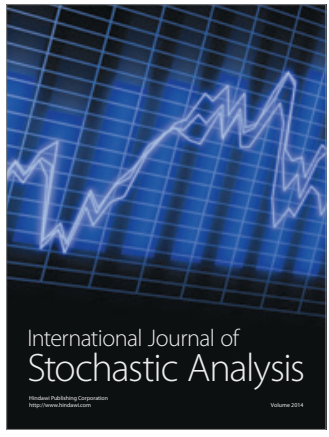

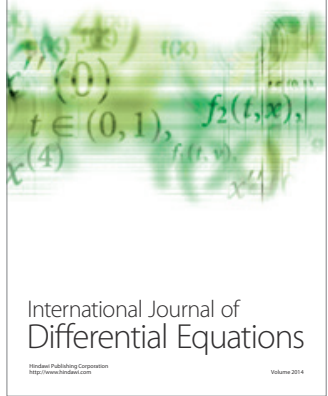
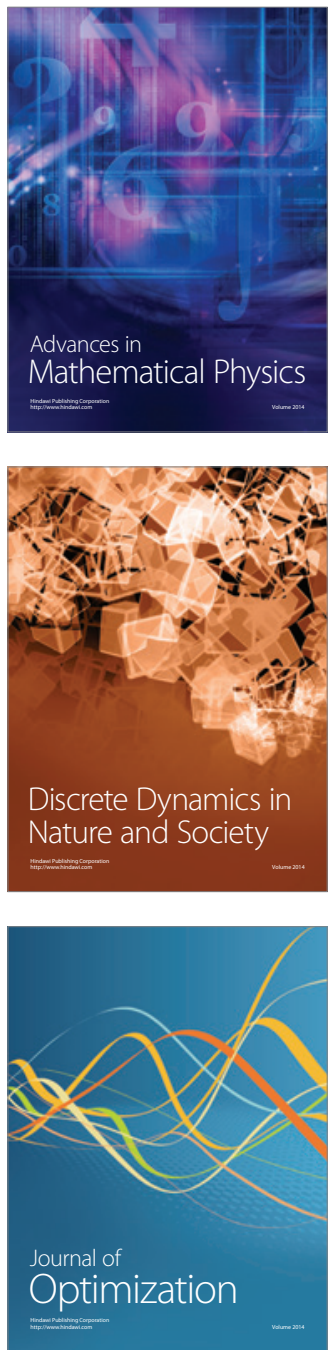